\title{
Skizze eines Verfahrens zur Erstellung von Ontologien mittels Formaler Begriffsanalyse
}

\author{
Christoph Lübbert ${ }^{1} \cdot$ Thomas Zeh $^{1}$ \\ Angenommen: 2. August 2021 / Online publiziert: 22. September 2021 \\ ๑ Der/die Autor(en) 2021
}

\section{Zusammenfassung}

Die Formale Begriffsanalyse vermag aus den Daten zu Gegenständen und deren Merkmalen eine Datenstruktur zu ermitteln. Es wird ein weitgehend automatisierbares Verfahren vorgestellt, das ausgehend von Elementarsätzen in Form von RDF-Tripeln in einem iterativen Bottom-up-Prozess mittels Semantischer Netze und der Formalen Begriffsanalyse zu einem Ontologieschema führt. Neben der Taxonomie der Klassen wird hierbei auch eine Taxonomie der Relationstypen erstellt. Die durch die Formale Begriffsanalyse automatisch erstellten Merkmalimplikationen können für die Qualitätssicherung herangezogen werden. Führt diese zu Korrekturen und Erweiterungen der Elementarsätze, wird der Prozess wiederholt. Das resultierende Ontologieschema wird in ein Entity-Relationship-Diagramm überführt, das die Grundlage für ein konzeptionelles Datenbankschema liefert. Zur Demonstration des Verfahrens wird exemplarisch eine Ontologie eines Wissensgebiets erstellt.

Alle Überlegungen zur Erstellung des Ontologieschemas lassen sich prinzipiell auch bei der Erstellung und Ausarbeitung beliebiger semantischer Datenschemata anwenden. Somit bietet diese Bottom-up-Methode die Chance, die Generierung des Datenbankschemas algorithmisch durch Beispielsammlungen zu unterstützen. Da jeder Schritt im Verfahren nachvollziehbar und somit von der Person des Modellierers unabhängig ist, liefert das Verfahren einen Beitrag zur Automatisierung der Modellierung, insbesondere von Ontologien.

\section{Motivation und Ziel}

Mit der Formalen Begriffsanalyse (FBA) [1] entfalten sich aus Daten zu Gegenständen und deren Merkmalen automatisch Begriffe, unter die diese Gegenstände fallen und die diese Merkmale beinhalten. Die FBA liefert somit anhand erhobener Daten eine Datenstruktur. Aus Daten lassen sich also Metadaten generieren, wie sie für semantische Datenmodelle von Wissensgebieten benötigt werden und die bei

\footnotetext{
Christoph Lübbert

christoph.luebbert@t-online.de

Thomas Zeh

thomas.zeh@t-online.de
}

1 Ernst-Schröder-Zentrum für Begriffliche Wissensverarbeitung, Fachbereich Mathematik, TU Darmstadt, Schlossgartenstr. 7, 64289 Darmstadt, Deutschland der Entwicklung von Ontologien [2] praktisch genutzt werden können. ${ }^{1}$

Anhand eines Beispiels wollen wir ein Verfahren aufzeigen, mit dem das Modellieren weitgehend automatisch, nachvollziehbar und somit objektivierbar und weniger personenabhängig gemacht werden kann. Der Artikel richtet sich vor allem an Informatiker und Linguisten und an Entwickler von Datenmodellen sowie an Wissensingenieure, die an einer Beschreibung von Weltausschnitten - sei es für die Schaffung von Anwendungssystemen, sei es für Ontologien - interessiert sind. ${ }^{2}$

\section{Aufgabe und Ausgangssituation}

Es gilt, für ein bestimmtes Wissensgebiet anhand von Aussagen über einige Instanzen ein zugehöriges Ontologie-

\footnotetext{
${ }^{1}$ Eine Aufstellung derartiger Verfahren findet sich bei Priya und $\mathrm{Ku}-$ $\operatorname{mar}[3]$.

${ }^{2}$ Der vorliegende Artikel ist eine Kurzfassung des mathematisch fundierten Artikels Ein Verfahren zur Erstellung von Ontologien mittels Formaler Begriffsanalyse von den gleichen Autoren [4].
} 
schema zu erstellen. Dies soll auf Basis einer Sammlung elementarer Sätze der Form Subjekt-Prädikat-Objekt (S-PO) geschehen. Die Elementarsätze liegen in Textform oder als RDF-Tripel [5] vor als sogenannte Statements oder Urteile, also für wahr gehaltene Aussagen. Um sachlogische Zusammenhänge zwischen den Termen der Elementarsätze grafisch darzustellen, wird ein Semantisches Netz genutzt.

\section{Formaler Kontext und Begriffsverband}

Nach DIN 2342 ist ein Begriff ,eine Denkeinheit, die aus einer Menge von Gegenständen unter Ermittlung der diesen Gegenständen gemeinsamen Eigenschaften mittels Abstraktion gebildet wird“. Der Gegenstandsbegriff wird hier sehr weit gefasst. Er umfasst nicht nur materielle und konkrete, sondern beliebige Dinge. Die formale Definition des Begriffs erfolgt in der Formalen Begriffsanalyse. Die FBA ist eine auf der Verbandstheorie basierende Methode zur Strukturierung von Daten mittels formaler Kontexte und deren Begriffsverbänden. Sie wurde in den 1980er-Jahren von Rudolf Wille, Bernhard Ganter und Peter Burmeister eingeführt.

Es folgen einige grundlegende Definitionen.

Gegeben sei eine Menge G, deren Elemente man Gegenstände nennt, und eine Menge $\mathrm{M}$, deren Elemente man Merkmale nennt, sowie eine Relation $\mathrm{I} \subseteq \mathrm{G} \times \mathrm{M}$, die man Inzidenzrelation nennt. Das Tripel $\mathrm{K}=(\mathrm{G}, \mathrm{M}, \mathrm{I})$ wird dann als formaler Kontext bezeichnet. Für einen Gegenstand $\mathrm{g} \in \mathrm{G}$ und ein Merkmal $\mathrm{m} \in \mathrm{M}$ liest man $(\mathrm{g}, \mathrm{m}) \in \mathrm{I}$ als: der Gegenstand $g$ hat das Merkmal $m$. Ein formaler Kontext (G, M, I) lässt sich in Form einer Kreuzchentabelle darstellen: Trifft $(\mathrm{g}, \mathrm{m}) \in \mathrm{I}$ zu, so bekommt die Zelle $(\mathrm{g}, \mathrm{m})$ der G-M-Tabelle (Inzidenzmatrix) ein Kreuzchen $\otimes$, andernfalls bleibt sie leer.

Ist $\mathrm{K}=(\mathrm{G}, \mathrm{M}, \mathrm{I})$ ein formaler Kontext und $\mathrm{A} \subseteq \mathrm{G}$, so ist die Menge der Merkmale, die alle Gegenstände von A gemeinsam haben

$A \uparrow:=\{m \in M \mid \forall g \in A:(g, m) \in I\}$.

Ist $\mathrm{B} \subseteq \mathrm{M}$, so ist die Menge der Gegenstände, die alle Merkmale von $\mathrm{B}$ gemeinsam haben

$B \downarrow:=\{g \in G \mid \forall m \in B:(g, m) \in I\}$.

Die Operatoren $\uparrow$ und $\downarrow$ nennt man Ableitungen im Kontext K. Durch sie werden Gegenstandsmengen auf Merkmalsmengen und umgekehrt abgebildet.
Ein Paar (A, B) heißt ein formaler Begriff im formalen Kontext $\mathrm{K}=(\mathrm{G}, \mathrm{M}, \mathrm{I})$, wenn gilt

$A \subseteq G, B \subseteq M, A \uparrow=B$ und $B \downarrow=A$.

Dabei heißt A der Umfang, B der Inhalt des formalen Begriffs (A, B). Die Menge aller formalen Begriffe des formalen Kontextes $\mathrm{K}$ wird mit $\mathfrak{B}(\mathbf{K})$ bezeichnet. Auf $\mathfrak{B}(\mathbf{K})$ führt man eine Ordnung (reflexive, antisymmetrische, transitive binäre Relation) ein durch

$$
(A, B) \leq\left(A^{\prime}, B^{\prime}\right): \Leftrightarrow A \subseteq A^{\prime}
$$

(und das gilt genau dann, wenn $B^{\prime} \subseteq B$ ).

Die Struktur $(\mathfrak{B}(\mathbf{K}), \leq)$ heißt der Begriffsverband des formalen Kontexts K, da diese Struktur mathematisch stets einen Verband darstellt.

Begriffsverbände können als Liniendiagramme dargestellt werden. Diese zeigen recht anschaulich die Daten in ihrer Struktur.

\section{Verfahren}

Mittels Semantischer Netze und der Formalen Begriffsanalyse wollen wir ein Verfahren vorstellen, das ausgehend von Elementarsätzen in einem iterativen Bottom-up-Verfahren zu einem Ontologieschema und einem Entity-RelationshipDiagramm (ERD) [6] führt. Zur Demonstration des Verfahrens erstellen wir exemplarisch und im Kleinen eine Ontologie eines Wissensgebiets. Wir wählen hierzu das Wissensgebiet Kunstgeschichte und verwenden das Beispiel aus dem Wikipedia-Artikel über Ontologie (Informatik) [7] in leicht modifizierter Form. Wir gehen von den Sachverhalten in der Instanzenebene (unterer Teil der Grafik in Abb. 1) aus und werden im Folgenden zeigen, dass das Ontologieschema (oberer Teil der Grafik) daraus prinzipiell ableitbar ist. Diese Ontologie kann u. a. der Erstellung eines Informationssystems für den an Kunstgeschichte interessierten Laien dienen.

Wir gehen von den folgenden 11 Aussagen aus:

- Santi malt Johannes den Täufer.

- Galleria dell'Accademia stellt Johannes der Täufer aus.

- Buonarroti schlägt den David.

- Galleria dell'Accademia stellt den David aus.

- Picasso malt den Jungen mit der Pfeife.

- Picasso ist Kubist.

- Santi ist Florentiner.

- Buonarroti ist Florentiner.

- Johannes der Täufer ist eine Ölzeichnung.

- Der Junge mit der Pfeife ist eine Ölzeichnung.

- David ist eine Steinskulptur. 
Abb. 1 Beispielontologie Kunstgeschichte mit Instanzenhttps://de.wikipedia.org/wiki/ Datei:Ontschichten.gif) und Schemaebene. (Mod. nach: Ontologie_(Informatik)\#/media/

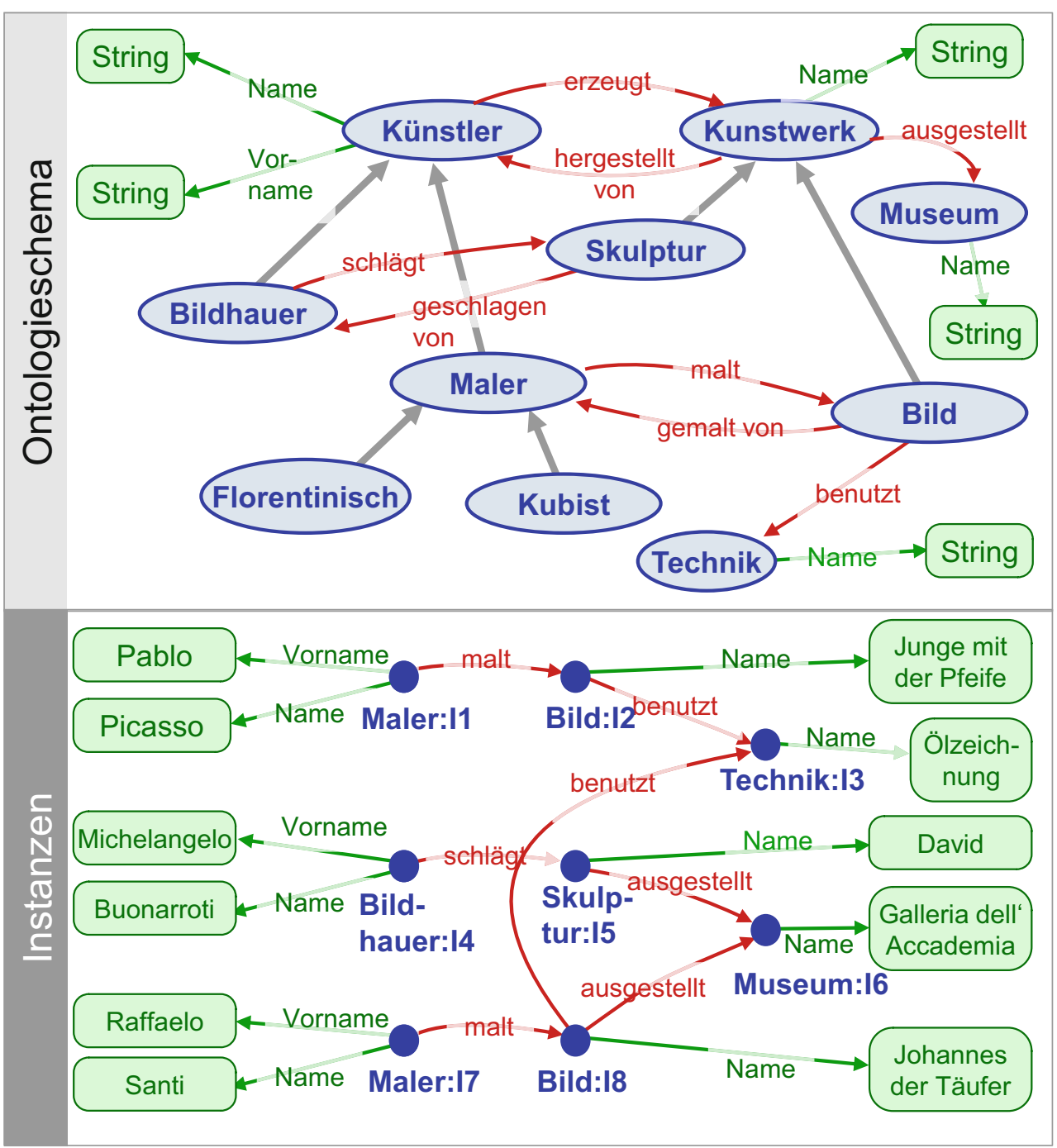

Abb. 2 Semantisches Netz SN1

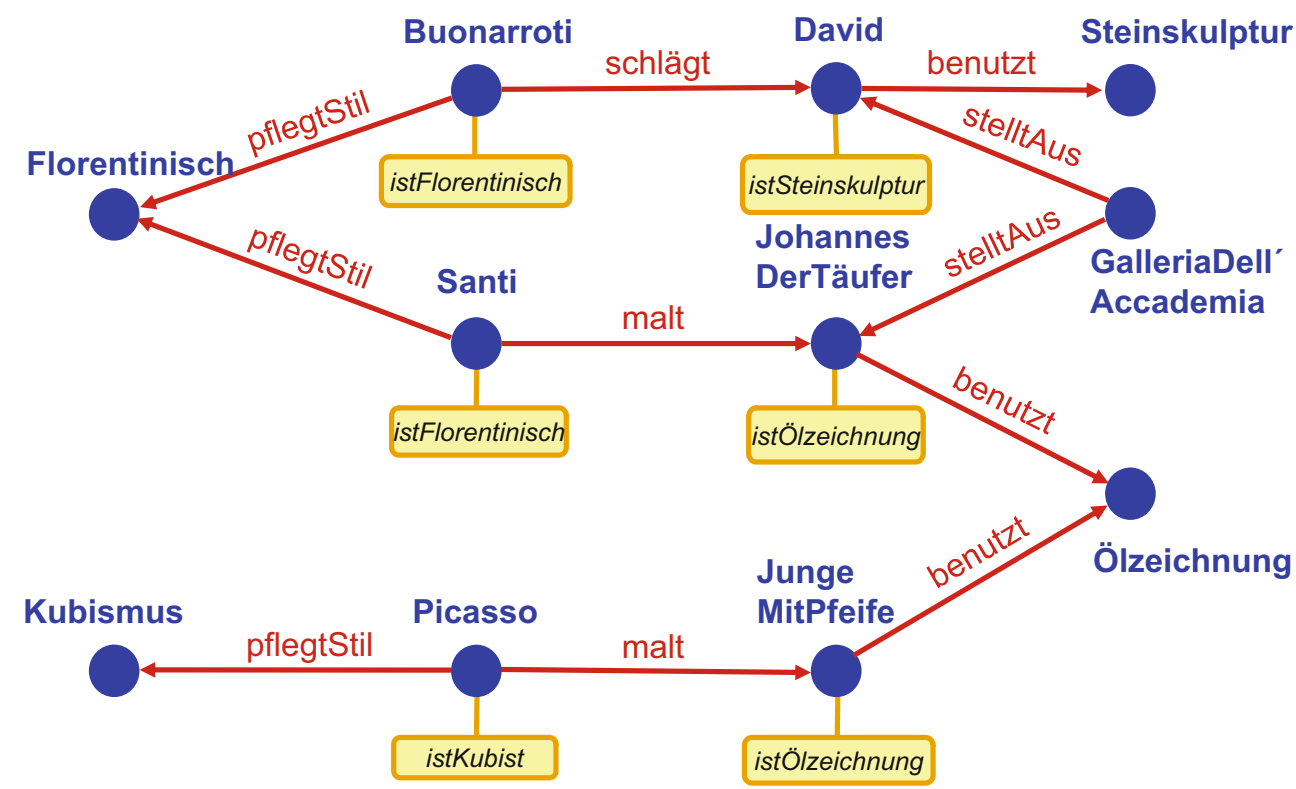


Abb. 3 Leitfaden zur Erstellung des Ontologieschemas

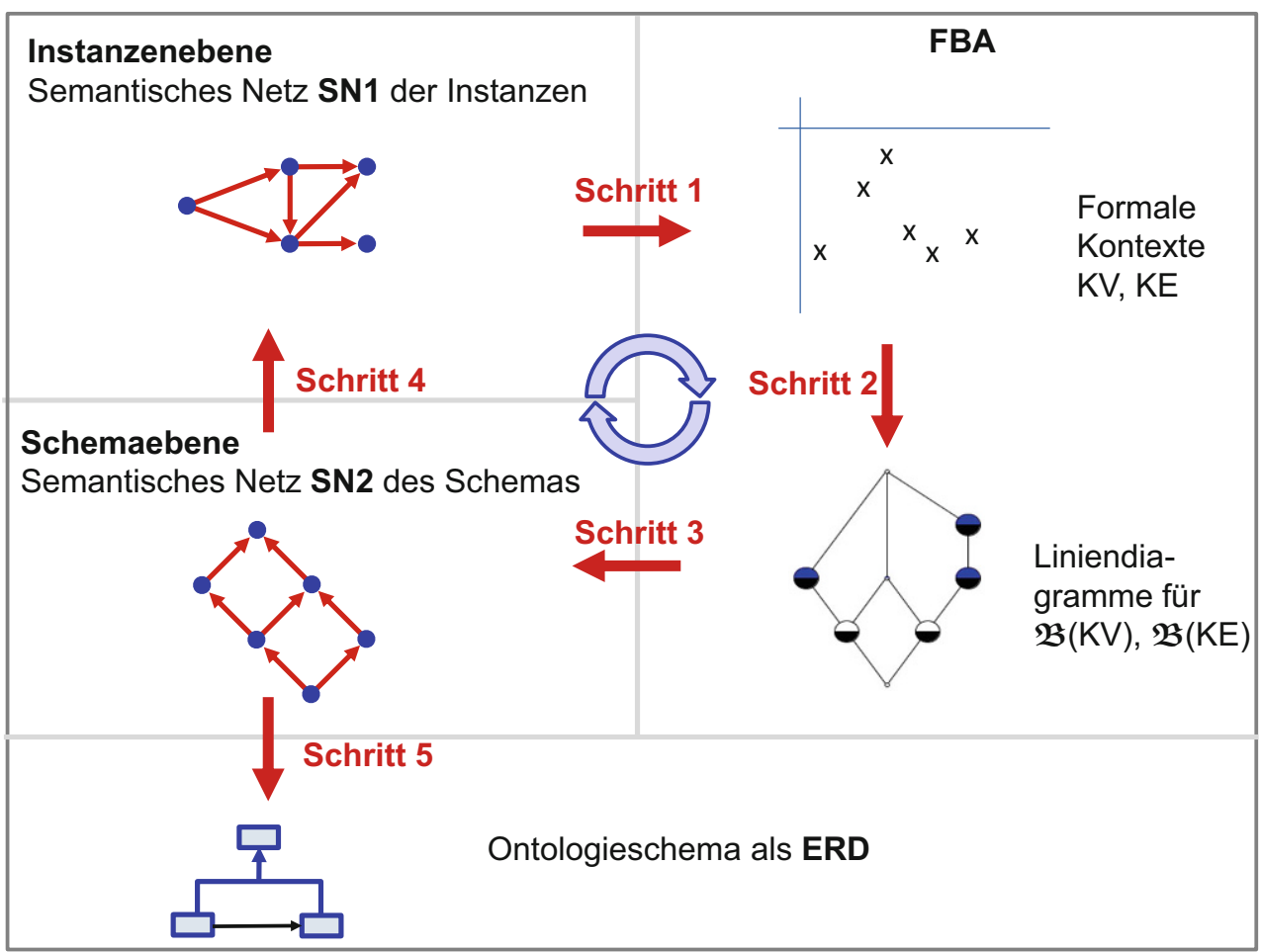

Diese Aussagen entsprechen denjenigen des WikipediaBeispiels. Bei den Künstlern haben wir die dort fehlenden Angaben zum Stil ergänzt. Anders als im Wikipedia-Beispiel gibt es hier auf der Instanzenebene nicht die Begriffe, wie z. B. Maler, Bild, Bildhauer, Skulptur und Museum. Wir werden zeigen, dass diese Begriffe erst anhand der Elementarsätze durch die FBA ermittelt resp. konstruiert werden und zur Metaebene, also zum Ontologieschema, gehören.

Es reicht dem Entwickler, wenn er einige Beispiele ermittelt hat und sich ein Bild von der ,strukturellen Breite“ der kunstgeschichtlichen Daten gemacht hat. Diese Sammlung von Elementarsätzen stellt die Ausgangsdatensituation dar. Sollten unter den Termen der Elementarsätze Synonyma auftauchen, so werden diese durch den Entwickler bereinigt, indem er einen bevorzugten Term festlegt; Homonyme werden von ihm berücksichtigt, indem er sie aufteilt und sie unterschiedlich benennt.

Basierend auf diesen Elementarsätzen erstellt der Entwickler ein Semantisches Netz. Hierbei wird jeder Elementarsatz der Form S-P-O auf ein Knotenpaar und eine gerichtete Kante überführt. Ein Elementarsatz kann auch zu einem Attribut des durch das Subjekt bestimmten Knotens führen. Die Entscheidung ob Strukturierung mittels Kanten oder Knotenattributierung ist in dieser Phase der Ontologieentwicklung meist willkürlich. Beispiel: „Picasso ist Kubist. " Soll dieser Elementarsatz als Beziehung pflegtStil zwischen den Knoten Picasso und Kubismus oder als Attribut istKubist von Picasso modelliert werden? Da beide
Varianten korrekt sind, modellieren wir sie nach der Devise nicht entweder-oder sondern sowohl-als auch.

Am einfachen Beispiel der Abb. 2 wollen wir demonstrieren, wie man, ausgehend von einer Sammlung von Elementarsätzen, mithilfe von FBA-Methoden zur Darstellung eines Ontologieschemas gelangt. Die Elementarsätze werden in das Semantische Netz SN1 überführt. Dessen Knoten, Knotenattribute und Kanten werden im Schritt 1 als formale Kontexte der Knoten KV und der Kanten KE in zwei Tabellen übertragen. Aus diesen Daten der Instanzenebene werden im Schritt 2 zwei Liniendiagramme mit den durch die FBA ermittelten Begriffen generiert, die der Schemaebene zuzurechnen sind. Sie werden im Schritt 3 in das Semantische Netz SN2 der Klassen überführt. Das Ontologieschema SN2 wird im Rahmen der Qualitätssicherung überprüft, was im Schritt $4 \mathrm{zu}$ einer Erweiterung und Korrektur der Elementarsätze und somit des Semantischen Netzes SN1 der Instanzen führen kann. In diesem Fall erfolgen die Schritte 1-4 zyklisch. Unser iteratives Bottomup-Verfahren endet, sobald das Ontologieschema alle Kriterien der Qualitätsprüfung erfüllt. Dann kann im Schritt 5 aus SN2 das ERD abgeleitet werden, das die Grundlage für ein Datenbankschema zur Erstellung der Datenbasis für die Ontologie ist.

Das Vorgehen aus den genannten fünf Schritten ist in Abb. 3 dargestellt. 


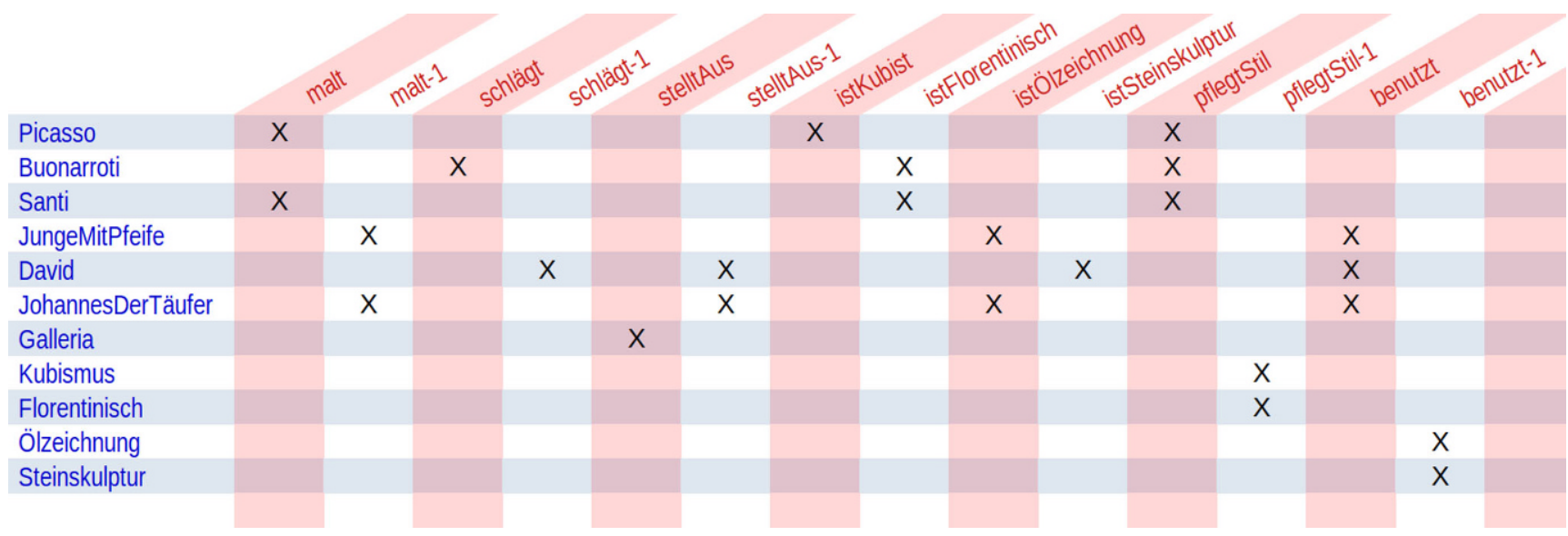

Abb. 4 Der Knotenkontext KV

\section{Schritt 1: Erstellung der formalen Kontexte zum Semantischen Netz der Instanzen}

Zum vorgegebenen SN1 der Abb. 2 bilden wir die Kreuzchentabelle des Knotenkontexts KV, wobei die Knoten die Gegenstände sind. Merkmale sind die Knotenattribute sowie die Beziehungen, an denen ein Knoten in einer bestimmten Rolle - als Subjekt oder als Objekt - beteiligt ist $\mathrm{zu}$ anderen Knoten wie auch ggf. zu sich selbst (Abb. 4).

Hiernach bilden wir zum vorgegebenen SN1 der Abb. 2 die Kreuzchentabelle des Kantenkontexts KE, ${ }^{3}$ wobei die gerichteten Kanten in beide Richtungen die Gegenstände und die Beziehungstypen die Merkmale sind. Die inverse Richtung eines Beziehungstyps wird durch ein nachgestelltes -1 gekennzeichnet (Abb. 5).

\section{Schritt 2: Erstellung der Begriffsverbände zu den formalen Kontexten}

Mit einem bewährten FBA-Tool [10] erzeugen wir aus dem Knotenkontext KV das Liniendiagramm des zugehörigen Begriffsverbands $\mathfrak{B}(\mathbf{K V})$ - siehe Abb. 6. Die Kreise im Liniendiagramm repräsentieren die Begriffe als Paare von Gegenstands- und Merkmalsmengen. Die Gegenstandsmenge eines Begriffs finden wir anhand der Gegenstandsnamen, die unter all den Kreisen stehen, die ausgehend von dem zum Begriff gehörenden Kreis durch einen absteigenden

\footnotetext{
${ }^{3}$ Elemente von Relationen als Gegenstände eines formalen Kontexts zu betrachten, ist eine Anwendung der von Rudolf Wille eingeführten power context families $(\mathrm{PCF})$, die er wie folgt definiert hat: Eine power context family ist eine Folge $\mathrm{K} !:=\left(\mathrm{K}_{0}, \mathrm{~K}_{1}, \mathrm{~K}_{2}, \ldots\right)$ von formalen Kontexten $\mathrm{K}_{\mathrm{k}}:=\left(\mathrm{G}_{\mathrm{k}}, \mathrm{M}_{\mathrm{k}}, \mathrm{I}_{\mathrm{k}}\right)$ mit $\mathrm{G}_{\mathrm{k}} \subseteq\left(\mathrm{G}_{0}\right)^{\mathrm{k}}$ für $\mathrm{k}=1,2, \ldots$ [8] Die formalen Gegenstände von $K_{k}$ mit $k=1,2, \ldots$ repräsentieren Elemente k-stelliger Relationen. In unserer Situation haben wir es neben $\mathrm{K}_{1}$ mit $\mathrm{K}_{2}$, also binären Relationen, zu tun. Bei der Überführung der semantischen Netze in PCFs wurde von uns der von Eklund und Groh in [9] beschriebene Algorithmus modifiziert.
}

Streckenzug erreichbar sind. Entsprechend finden wir die Merkmalsmenge eines Begriffs durch die über den Kreisen stehenden Merkmalsnamen aller aufsteigenden Streckenzüge. Die Verbandsordnung von $\mathfrak{B}(\mathbf{K V})$ ist später im Schritt 3 als die Klassentaxonomie im Ontologieschema zu interpretieren.

Entsprechend erzeugen wir aus dem Kantenkontext KE das Liniendiagramm des zugehörigen Begriffsverbands $\mathfrak{B}(\mathbf{K E})$. Die Verbandsordnung von $\mathfrak{B}(\mathbf{K E})$ ist $\mathrm{zu}$ interpretieren als die Taxonomie der Relationstypen im Ontologieschema, die hier zunächst noch flach ist.

\section{Schritt 3: Erstellung des Ontologieschemas}

Das Liniendiagramm von $\mathfrak{B}(\mathbf{K V})$ in der Abb. 6 zeigt im Wesentlichen bereits das Grundgerüst des Ontologieschemas. Die durch die Kreise repräsentierten Begriffe im Liniendiagramm haben noch keine Bezeichner. Die Namensvergabe setzt einen mentalen Akt voraus. Es ist nicht damit getan, dem Knoten ein „Etikett zu verpassen“, sondern es gilt für den Experten des Wissensgebiets anhand der Gegenstandsmenge und der Merkmalsmenge eines jeden Begriffs im Begriffsverband einen entsprechenden Begriff aus dem zu modellierenden Wissensgebiet ausfindig zu machen. Dieser muss alle Gegenstände der Gegenstandsmenge umfassen und alle Merkmale der Merkmalsmenge aufweisen. Die Bezeichnung dieses so gefundenen Begriffs kann dann als Klassenname herangezogen werden.

Nach der Vergabe sprechender Klassennamen folgt die Einbettung der Relationstypen. Hierfür sind für einen Relationstyp $r$ die jeweils zwei Klassen ausfindig zu machen, zwischen denen der Relationstyp ,,aufgehängt" wird, d.h. es gilt, die zwei vom Umfang kleinsten Klassen zu ermitteln, zwischen deren Instanzen die Beziehung besteht.

Dazu helfen die Ableitungsoperatoren $\uparrow$ und $\downarrow$ der FBA wie folgt: 
Abb. 5 Der Kantenkontext KE

Abb. 6 Der Begriffsverband $\mathfrak{B}(\mathrm{KV})$
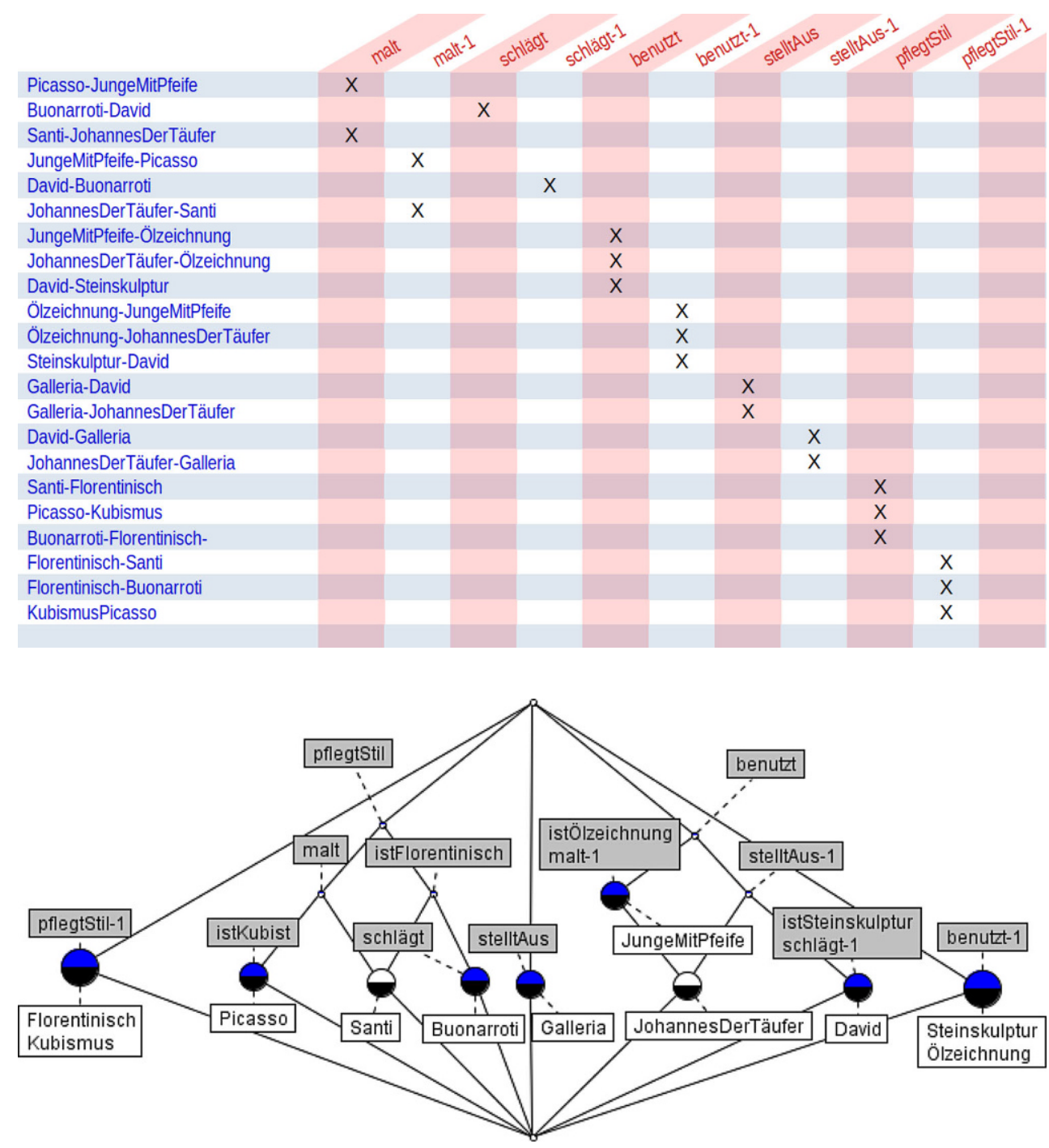

Ist A eine Menge an Gegenständen, zu der der Begriff mit dem kleinsten Umfang $U$ gesucht wird, der alle Elemente von A enthält, dann ist $\mathrm{U}=(\mathrm{A} \uparrow) \downarrow$ die Gegenstandsmenge, die zum gesuchten Begriff gehört. Dieser eindeutig bestimmte Begriff entspricht dann der gesuchten Klasse. Damit können wir das Ontologieschema mit den Relationstypen als Semantisches Netz SN2 erstellen (Abb. 7).

\section{Schritt 4: Überprüfung des Ontologieschemas}

Die im Semantischen Netz SN1 abgebildeten Elementarsätze können durch unzureichende Erhebung unvollständig oder unzutreffend sein. So fällt z.B. auf, dass die Klasse Skulptur Unterklasse der Klasse Ausstellungsobjekt ist; d. h. jede Skulptur wird ausgestellt. Der Kunstkenner weiß allerdings von Fällen, dass es auch Skulpturen gibt, die nicht ausgestellt werden, so z.B. die Skulptur Reclining Figure des Naturalisten Henry Moore. Dies führt zu den Elementar- sätzen „Henry Moore schlägt Reclining Figure.“, „Henry Moore ist Naturalist. “ und „Reclining Figure ist eine Bronzeskulptur.".

Der Schritt 4, also die Überprüfung des Ontologieschemas, kann nicht automatisiert werden. Allenfalls können bei der Überprüfung des Ontologieschemas mithilfe der Merkmalimplikation (Schlussfolgerungen zwischen Merkmalen des formalen Kontexts), deren automatisch durch das FBA-Programm ConExp erstellten Implikationen als Stütze dienen. Derartige Implikationen zwischen Merkmalen sind im vorliegenden Kontext beispielsweise:

$$
\text { istKubist } \rightarrow \text { malt } \text { und } \text { istNaturalist } \rightarrow \text { schlägt. }
$$

Lehnt der Experte eine Implikation ab, dann ist er gefordert, einen neuen Elementarsatz zu ergänzen (wie es bei der obigen Klasse Skulptur der Fall war) oder ggf. einen alten - dann falschen - zu streichen. Um die zweite der beiden o. a. Implikationen zu widerlegen, reicht es aus, einen neu- 
Abb. 7 Ontologieschema als Semantisches Netz SN2

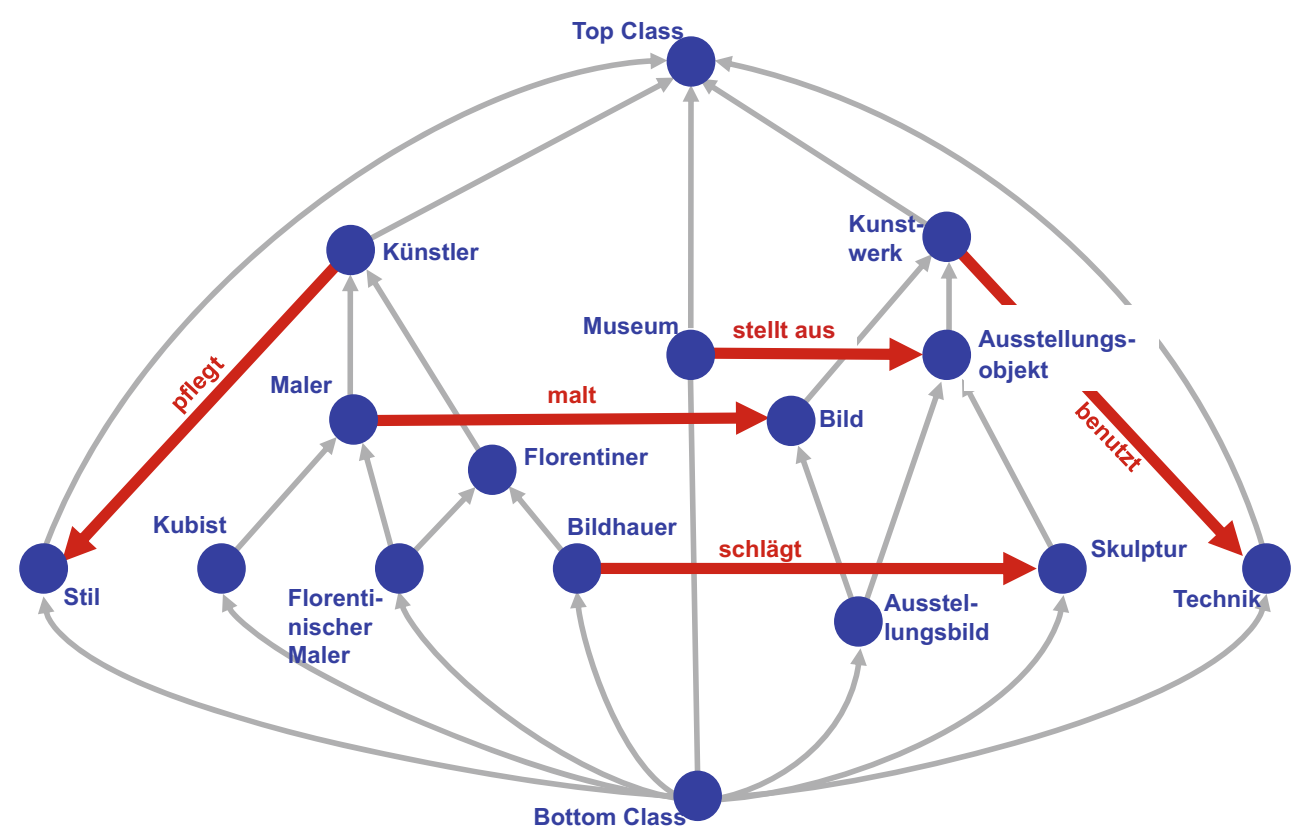

Legende

$\longrightarrow$ : is-a-Beziehung zur Darstellung der Klassentaxonomie

: Relationstyp
Abb. 8 Begriffsverband der Knoten $\mathfrak{B}(\mathrm{KV})$ nach dem zweiten Durchlauf

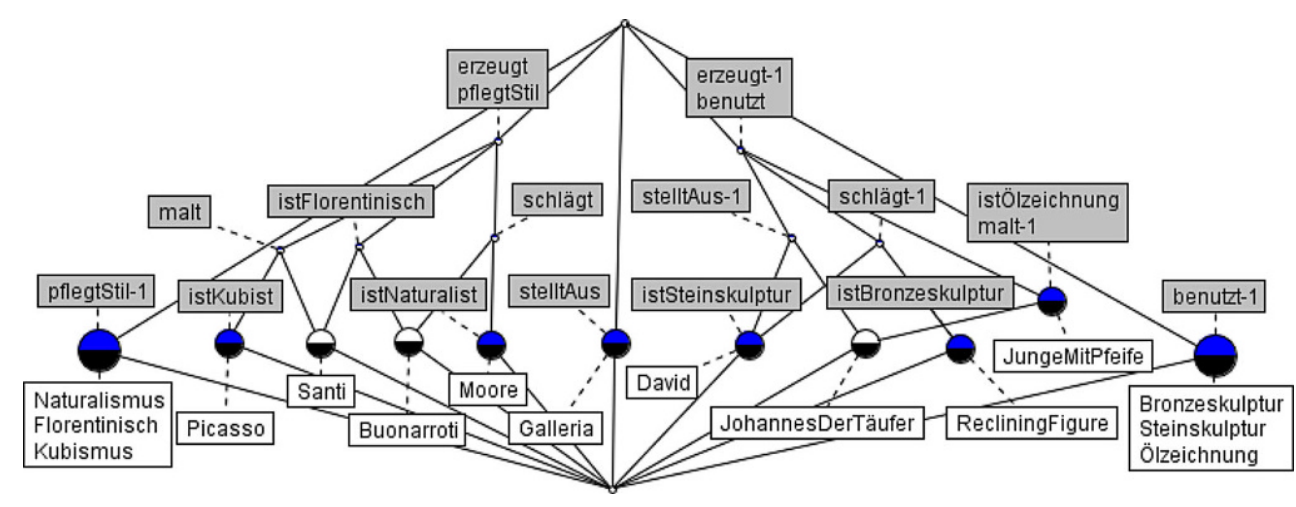

en Elementarsatz mit einem Naturalisten aufzunehmen, der keine Skulpturen schlägt (Abb. 8).

Die FBA liefert im Liniendiagramm der Kanten die Relationstypen-Taxonomie, welche bei der konventionellen Top-down-Methode meist nicht erkannt oder berücksichtigt wird. In unserem Fallbeispiel war sie nach dem ersten Durchgang trivial; die Hierarchie war flach. Nach dem zweiten Durchlauf ist das Ergebnis wie folgt (Abb. 9).

Als Ergebnis des 2. Durchlaufs erhalten wir das folgende Klassendiagramm (Abb. 10).

\section{Schritt 5: Ableitung des Entity-Relationship- Diagramms}

Das Ontologieschema in Abb. 10 kann in ein ERD ${ }^{4}$ überführt werden, wobei nicht alle Knoten und Kanten des SN2 in das ERD übernommen werden. Kandidaten für Entitätstypen sind solche Klassen, für die es betriebliche Funktionen gibt, die sie auch verwenden, und Klassen, die an Relationstypen beteiligt sind. Im ERD wird zur Darstellung der Kardinalitäten die (min, max)-Notation verwendet. Die einzelnen Häufigkeitsangaben werden dem aktuellen Datenbestand entnommen. Sie sind bei der Qualitätssicherung zu überprüfen und deren Ergebnis kann genutzt werden, um

${ }^{4}$ Zur Visualisierung des Ontologieschemas kann auch ein UML-Datenstrukturdiagramm genutzt werden. 


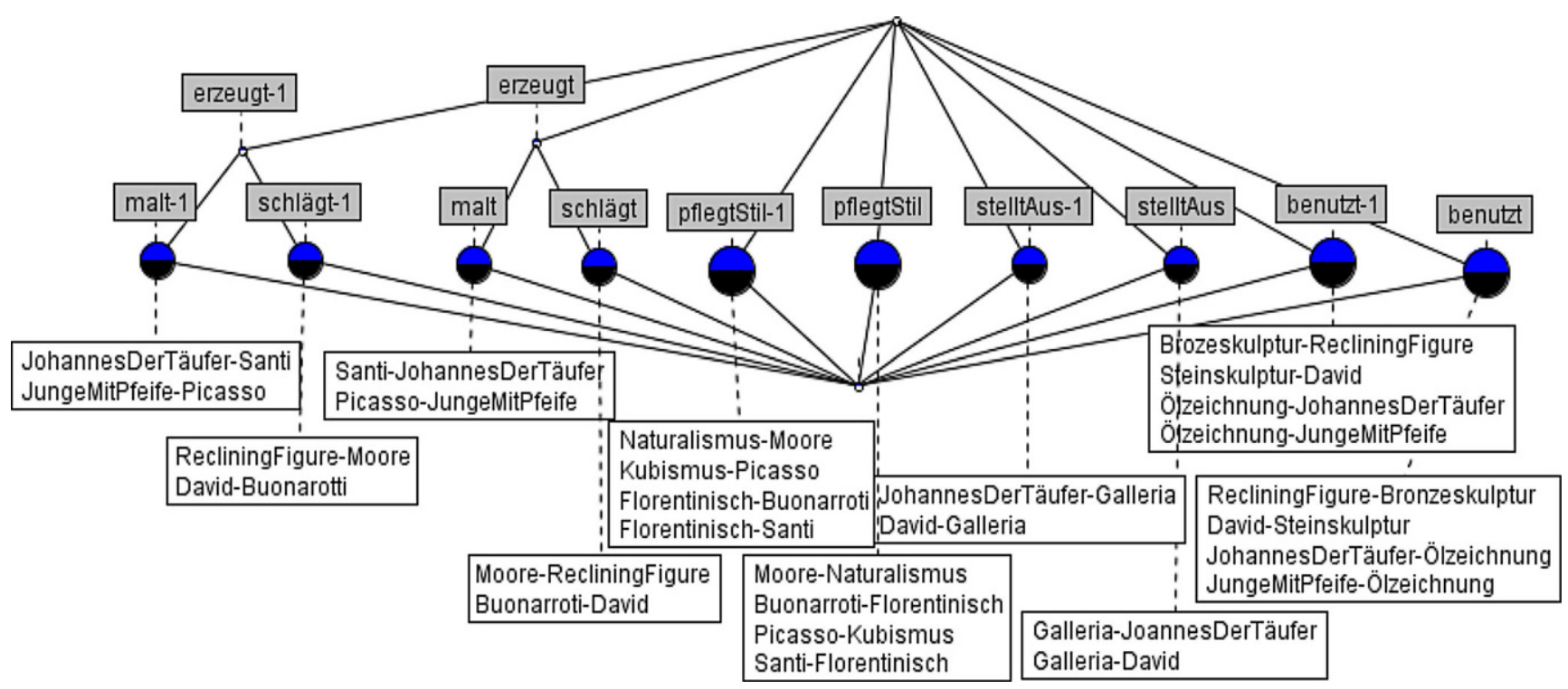

Abb. 9 Begriffsverband der Kanten $\mathfrak{B}(\mathrm{KE})$ nach dem zweiten Durchlauf

Abb. 10 Ontologieschema als Semantisches Netz SN2 nach dem zweiten Durchlauf

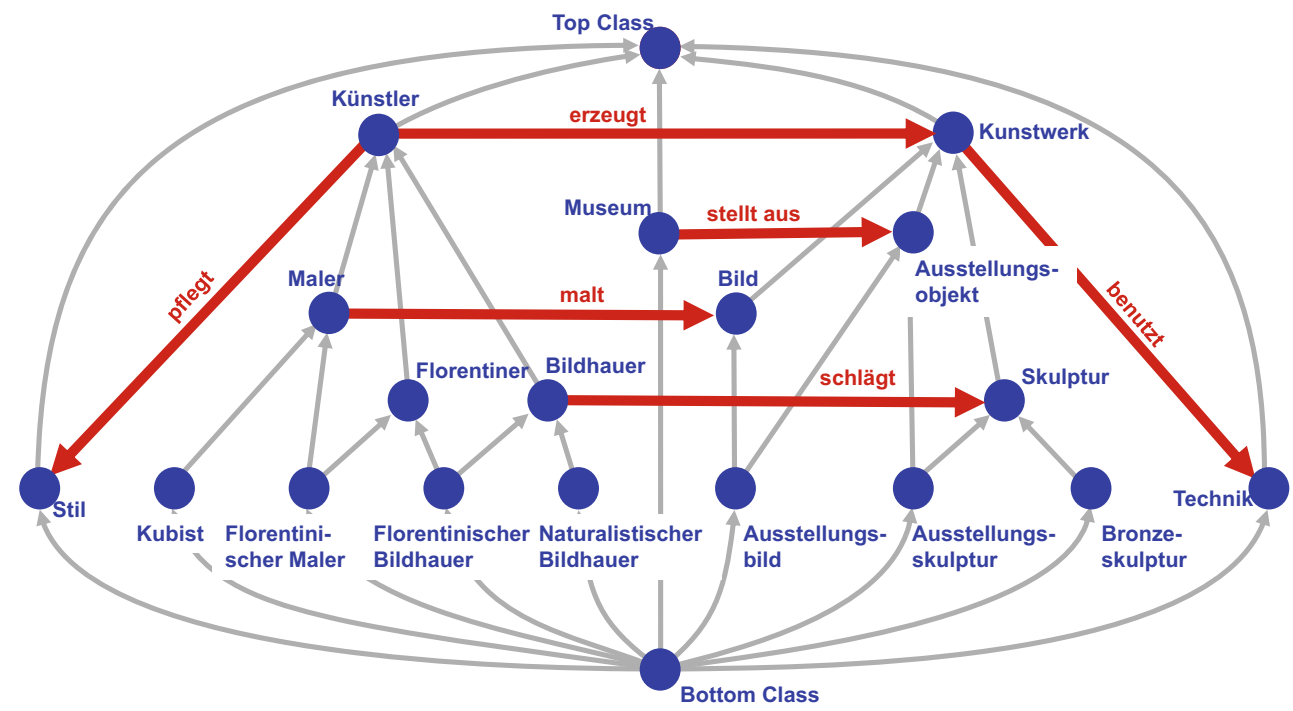

Legende das Datenmodell realitätsgetreuer zu gestalten. So kann im Beispiel mit dem Datenbestand nach dem zweiten Durchlauf gefragt werden, ob es zutrifft, dass ein Bildhauer genau eine Skulptur schlägt, und ob nicht ein Künstler mehrere Stile pflegen kann (Abb. 11).

Im ERD lässt sich die Taxonomie der Relationstypen nicht ohne weiteres darstellen.

Die Implikationen

malt $\rightarrow$ erzeugt und schlägt $\rightarrow$ erzeugt können aber als Integritätsbedingungen in das konzeptionelle Datenmodell eingehen und in der Ontologie neben den Merkmalimplikationen die Inferenzmaschine anreichern.

Ein Attribut einer Klasse wird demjenigen Entitätstyp zugeordnet, der innerhalb der Entity-Relationship-Ordnungsstruktur von unten nach oben gehend erstmalig alle Entitäten mit den zugehörigen Attributwerten umfasst.

Aus dem ERD lässt sich dann das konzeptionelle Datenbankschema für die Ontologie ableiten. 
Abb. 11 Entity-RelationshipDiagramm mit der (min, max)Notation

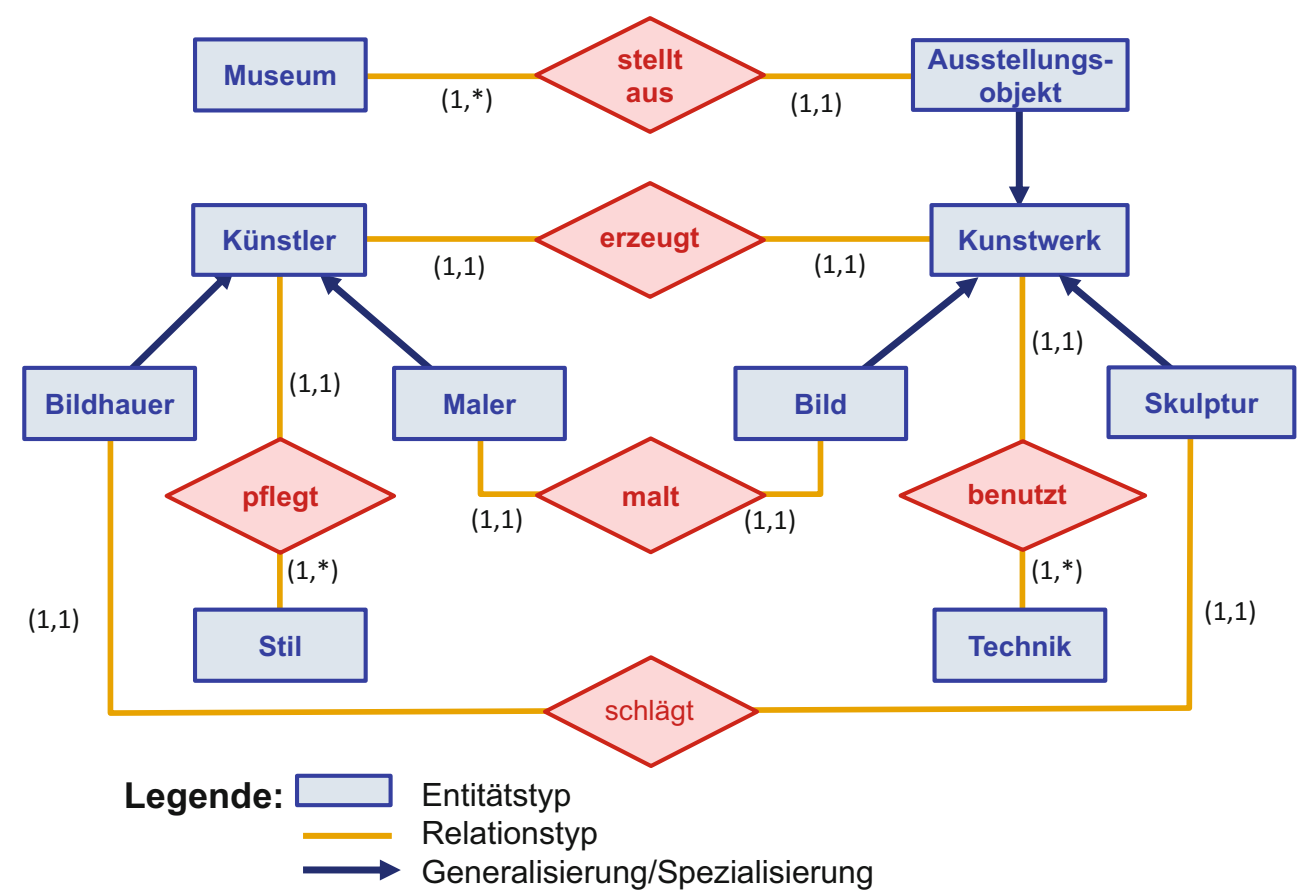

\section{Potenziale/Nutzenaspekte}

Wir sehen in dem weitgehend automatisierbaren Verfahren eine Möglichkeit, den Modellierungsprozess und die Qualitätssicherung zu unterstützen, sowie einen Beitrag zur Konsensfindung bei den an der Entwicklung von Ontologien Beteiligten. Die Liniendiagramme zum Knoten- und Kantenkontext, das Semantische Netz des Ontologieschemas wie auch die automatisch erkannten logischen Zusammenhänge durch die Merkmalimplikation werfen inhaltliche Fragen auf und unterstützen damit den Entwickler und den Qualitätssicherer zielführend bei der Konzeption des Ontologieschemas. Auch ist jeder Schritt im Verfahren nachvollziehbar und somit von der Person des Modellierers unabhängig.

Alle Überlegungen zur Erstellung des Ontologieschemas lassen sich prinzipiell auch bei der Erstellung und Ausarbeitung beliebiger semantischer Datenschemata anwenden. Somit bietet diese Bottom-up-Methode die Chance, die Generierung des Datenbankschemas algorithmisch durch Beispielsammlungen zu unterstützen. Auch kann ein auf anderem Weg erstelltes semantisches Datenschema mit dem Ergebnis des FBA-basierten Verfahrens verglichen werden, um von den Widersprüchen wie auch den strukturellen Ergänzungen $-z$. B. durch neue Begriffe oder Beziehungen zu profitieren.

Da uns nur einige Instanzen aus der Datenbasis zum Ontologieschema führen, das für alle Daten des Wissensgebietes gelten soll, muss das induktiv entstandene Ergebnis durch weitere Elementarsätze auf Plausibilität überprüft werden. Dies kann zu einem Prozess ausgebaut werden, in- dem neue Instanzen zu gegebenen Zeiten gegen das Schema laufen, das dann im Konfliktfall differenziert und damit erweitert bzw. um strukturelle Neuheiten ergänzt wird. Damit können die Erweiterung eines Wissensgebietes und auch ein Strukturwandel in der Ontologie einfacher berücksichtigt werden. Überträgt man diesen Prozess auf zwei oder mehr bestehende Ontologien, so kann das Verfahren auch zur Schemaintegration der Ontologien, basierend auf Auszügen aus deren Datenbeständen, genutzt werden. ${ }^{5}$

Funding Open Access funding enabled and organized by Projekt DEAL.

Open Access Dieser Artikel wird unter der Creative Commons Namensnennung 4.0 International Lizenz veröffentlicht, welche die Nutzung, Vervielfältigung, Bearbeitung, Verbreitung und Wiedergabe in jeglichem Medium und Format erlaubt, sofern Sie den/die ursprünglichen Autor(en) und die Quelle ordnungsgemäß nennen, einen Link zur Creative Commons Lizenz beifügen und angeben, ob Änderungen vorgenommen wurden.

Die in diesem Artikel enthaltenen Bilder und sonstiges Drittmaterial unterliegen ebenfalls der genannten Creative Commons Lizenz, sofern sich aus der Abbildungslegende nichts anderes ergibt. Sofern das betreffende Material nicht unter der genannten Creative Commons Lizenz steht und die betreffende Handlung nicht nach gesetzlichen Vorschriften erlaubt ist, ist für die oben aufgeführten Weiterverwendungen des Materials die Einwilligung des jeweiligen Rechteinhabers einzuholen.

Weitere Details zur Lizenz entnehmen Sie bitte der Lizenzinformation auf http://creativecommons.org/licenses/by/4.0/deed.de.

\footnotetext{
${ }^{5}$ Einen FBA-basierenden Bottom-up-Ansatz für den Merge-Prozess von Ontologien verfolgen auch Stumme und Maedche in [11].
} 


\section{Literatur}

1. Ganter B, Wille R (1996) Formale Begriffsanalyse (FBA). Springer, Berlin, Heidelberg

2. Busse J, Humm B, Lübbert C, Moelter F, Reibold A, Rewald M, Schlüter V, Seiler B, Tegtmeier E, Zeh T (2014) Was bedeutet eigentlich Ontologie? - Ein Begriff aus der Philosophie im Licht verschiedener Disziplinen. Informatik Spektrum 37(4):286-297

3. Priya M, Aswani Kumar C (2015) A survey of state of the art of ontology construction and merging using formal concept analysis. Indian J Sci Technol. https://doi.org/10.17485/ijst/2015/v8i24/82808

4. Lübbert C, Zeh T Ein Verfahren zur Erstellung von Ontologien mittels Formaler Begriffsanalyse (Publikation vorgesehen)

5. Barrasa J Field engineer: RDF triple stores vs. labeled property graphs: what's the difference? https://neo4j.com/blog/rdf-triple-storevs-labeled-property-graph-difference/

6. Elmasri R, Navathe SB (2015) Fundamentals of database systems. Addison Wesley, Boston
7. Wikipedia - Die freie Enzyklopädie Ontologie (Informatik). https:// de.wikipedia.org/wiki/Ontologie_\%28Informatik\%29. Zugegriffen: 30 Apr 2021

8. Wille R (1997) Conceptual graphs and formal concept analysis. In: Lukose D, Delugach H, Keeler M, Searle L, Sowa JF (Hrsg) Conceptual structures: fulfilling Peirce's dream. LNAI, Bd. 1257. Springer, Heidelberg, S 290-303

9. Groh B, Eklund P (1999) Algorithms for Creating Relational Power Context Families from Conceptual Graphs. In: Proceedings 7thInternational Conference on Conceptional Structures. LNAI, Bd. 1640, Springer, Heidelberg, S 389-400

10. Beschreibungen und Downloadmöglichkeiten von Software zur FBA. http://www.ernst-schroeder-zentrum.de/sw.html. Zugegriffen: 28. Apr. 2021

11. Stumme G, Maedche A (2001) FCA-merge: a bottom-up approach for merging ontologies. In: Proc. 17th Int'l Joint Conf. Artificial Intelligence IJCAI '01. Morgan Kaufmann, San Francisco 